\title{
Stoppers Energy Harvesting Efficiency Enhancement via Optimal Linear Controller
}

\section{Douglas da Costa Ferreira}

Department of Mechanical Engineering, Federal University of Mato Grosso, Rondonópolis, MT

\section{Fábio Roberto Chavarette ${ }^{2}$}

Department of Mathematics, UNESP - Univ. Estadual Paulista, Ilha Solteira, SP

\section{Jean-Marc Stephane Lafay ${ }^{3}$}

Department of Mechanical Engineering, Federal Technological University of Paraná, Pato Branco, PR

\section{Paulo Rogerio Novak ${ }^{4}$}

Department of Mechanical Engineering, Federal Technological University of Paraná, Pato Branco, PR

\section{Samuel Pagotto}

Department of Mechanical Engineering, Federal University of Mato Grosso, Rondonópolis, MT

\section{Vinícius Favretto ${ }^{6}$}

Department of Mechanical Engineering, Federal University of Mato Grosso, Rondonópolis, MT

\begin{abstract}
A stoppers solution to enhance energy harvesting imposes a nonlinear behavior to the system and also a chaotic behavior that increase the mechanical energy transferring from excitation source to electric output power. To increase even more energy conversion it was project an Optimal Linear Control to drive the system to a optimize interaction from vibration source to structural system. A piezoelectric film is attached to a cantilever beam and an electric output load is assembled to extract electric energy from vibration sourced. The stoppers are arranged to improve the system efficiency. Using the indirect piezoelectric effect a controller will insert velocity and acceleration to the system and as main result the system increased efficiency 1.18 times considering output voltage rate.
\end{abstract}

Key-words. Energy harvesting, Efficiency, Optimal Linear Control, Stoppers, Impulse

\footnotetext{
1 dcferreira@ufmt.br

2 fabioch@mat.feis.unesp.br

3 jeanmarc@utfpr.edu.br

4 novak@utfpr.edu.br

5 samupagotto@gmail.com

6 vinicius_favreto@hotmail.com
} 


\section{Introduction}

An important promise solution to clean and sustainable energy source are the energy harvesting systems. To harness energy from small sources as fell gradients of temperature, sound, radio frequency and vibration [12] can be accomplished via electrostatic, electromagnetic and piezoelectric means [8] and a piezoelectric based energy harvesting subject to a vibration sourced is the focusing of this research. A piezoelectric material in direct effect behavior converts mechanical energy into electricity and an energy harvesting system can utilizes vibration as source to supply electrical power [6] to a small electronic device without necessity of a physical connection to any other electrical source. Most of actual research of vibration based energy harvesting are focusing in resonant solution $[15,9,11]$ were natural frequency is coincident to excitation vibration frequency. Nevertheless ambient vibration is wide band [5] and resonant effect occurs only for a narrow space of time and the efficiency of this solution remains insufficient for his proposes [16]. A nonlinear solution proposed by [2] increase efficiency by rising relation from ambient excitation and energy harvesting system response. Although non-linear has appeared as a better solution it is even insufficient to several applications regarding electrical consumption necessities [16]. A possible solution to enhance energy harnessing can be accomplished by controlled system projects that can improve interaction between excitation source and energy harvesting system vibration [14]. An Optimal Linear Control (OLC) is a controller proposed by [7] that can drive a non-linear dynamical system. Applications of OLC to non-linear systems are accomplished by [13] and specifically applications of OLC to enhancement of energy harvesting systems efficiency were studied by [3]. A stoppers energy harvesting system was proposed by [4] as shown in Figure 1. The experiment presented a significant increase of energy due impact effect. The use of impact as a mean to increase energy harvesting efficiency is a novel proposal that must be deeply explored. In this direction the present investigation will propose a control project based on OLC to a non-linear system to optimize the interaction between the vibration source and the harvesting system. The paper is organized in five chapters: energy harvesting system model, controller project, efficiency gain analysis and conclusions plus acknowledgements.

\section{Energy Harvesting System Model}

A stopper energy harvesting system utilizes the impact to enhance mechanical energy transfer from ambient vibration to the transduction system as shown in Figure 1.

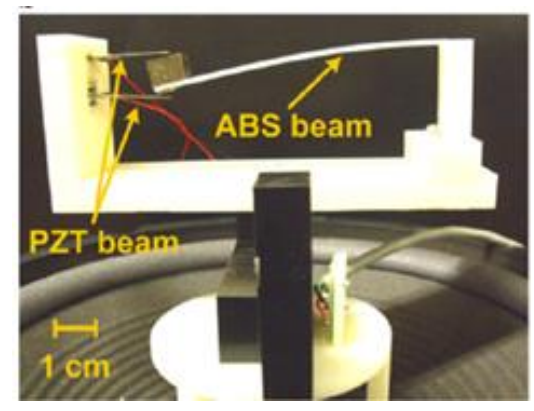

Figure 1: Stoppers energy harvesting system configuration [4]. 
3 The mathematical equations for impact energy harvesting system proposed by [4] is:

$$
\begin{array}{ll}
m \ddot{x}+c_{d} \dot{x}+k_{d} x=m a \sin \left(\omega_{d} t\right) & -d<x \\
m \ddot{x}+\left(c_{d}+c_{g 1}\right) \dot{x}+k_{d} x+k_{g 1}(x-d)=m a \sin \left(\omega_{d} t\right) & x \geq d \\
m \ddot{x}+\left(c_{d}+c_{g 2}\right) \dot{x}+k_{d} x+k_{g 2}(x+d)=m a \sin \left(\omega_{d} t\right) & x \leq d
\end{array}
$$

were $m$ is the proof mass at the tip of the cantilever subject to an exogenous described by ma $\sin \left(\omega_{d} t\right)$ were $a$ is the excitation acceleration and $\omega_{d}$ is the excitation frequency. The damping (c) and the stiffness (k) are given according the driving bean $\left(\mathrm{c}_{\mathrm{d}}, \mathrm{k}_{\mathrm{d}}\right)$ and the generating bean $\left(\mathrm{c}_{\mathrm{g}}, \mathrm{k}_{\mathrm{g}}\right)$. There were two generating beans named bean 1 and bean 2 . The gap between the proof mass and the generating beans is $\mathrm{d}$. The time depending variables is $\mathrm{x}$ for tip cantilever position. According [4] when the displacement is inferior to the gap between the proof mass and the generation beams $(-\mathrm{d}<\mathrm{x}<\mathrm{d})$ there are no electrical generation. Otherwise when the displacement is superior to the gap the generations are given by the amount of displacement of the piezoelectric beam as $(x-d)$ for upper interference and $(x+d)$ for lower interference. As a result the impact energy harvesting sourced more mechanical energy to the system and consequently more output power as presented in Figure 2.

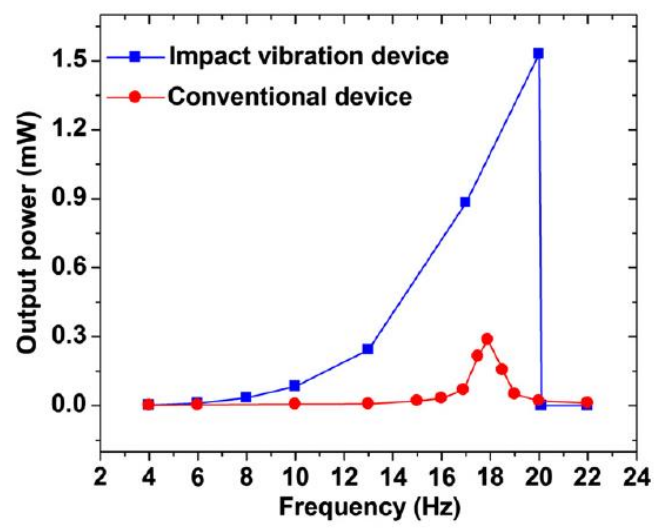

Figure 2: Comparison of impact and conventional energy harvest systems [4].

For numerical simulation it is proposed a comparison of a singular cantilever beam subjected to an exogenous excitation, dimensionless described by [2],

$$
\begin{aligned}
\ddot{x}+2 \zeta \dot{x}+\frac{1}{2} x-\chi v & =f \cos \Omega t \\
\dot{v}+\Lambda v+k \dot{x} & =0
\end{aligned}
$$

were $\zeta$ is damping factor, $\chi$ is piezoelectric mechanical coupling coefficient, $\Lambda$ is reciprocal of time constant to capacitive load and $\kappa$ is piezoelectric electric coupling coefficient. The excitation is described by $f$ as acceleration rate and $\Omega$ as frequency rate. The time depending variables are $x$ for tip cantilever position and $v$ for output voltage rate. Using a single stopper to produce impact to the system the induced behavior is approximate to a non-ideal power source as described by [10] and adopted as: 


$$
\begin{gathered}
\ddot{x}+2 \zeta \dot{x}+\frac{1}{2} x-\chi v=\dot{\varphi}^{2} \cos \varphi+\ddot{\varphi} \sin \varphi \\
\ddot{\varphi}=\ddot{x} \sin \varphi \\
\dot{v}+\Lambda v+\kappa \dot{x}=0
\end{gathered}
$$

were $\varphi$ is the phase angle of induced movement by impact force. Isolating $\ddot{x}, \ddot{\varphi}$ and $\dot{v}$ in Equation 3 and adopting $\mathrm{y}_{1}=x, y_{2}=\dot{x}, y_{3}=\varphi, y_{4}=\dot{\varphi}$ and $y_{5}=v$ the space-state of the dynamical system is given as:

$$
\begin{gathered}
\dot{y}_{1}=y_{2} \\
\dot{y}_{2}=\frac{-2 \zeta y_{2}-\frac{1}{2} y_{1}+\chi y_{5}+y_{4}{ }^{2} \cos y_{3}}{\left(1-\sin ^{2} y_{3}\right)} \\
\dot{y}_{3}=y_{4} \\
\dot{y}_{4}=\frac{\left(-2 \zeta y_{2}-\frac{1}{2} y_{1}+\chi y_{5}+y_{4}^{2} \cos y_{3}\right) \sin y_{3}}{\left(1-\sin ^{2} y_{3}\right)} \\
\dot{y}_{5}=-\kappa y_{2}-\Lambda y_{5}
\end{gathered}
$$

Applying Runge-Kutta forth order in equation (4) for dimensionless parameters $\zeta=$ $0.01, \Omega=0.8, \chi=0.05, \kappa=0.5, \Lambda=0.05$ and $f=0.083$ [2] and initial conditions $x(0)=$ $1, \varphi(0)=1, \dot{x}(0)=0, \dot{\varphi}(0)=0$ and $v(0)=0$ it is determined the voltage rate and the time history considering samples a from 0 to 2,500 in interval of 0.1 totalizing 25,000 time sample according Figure 3a: output voltage and $3 \mathrm{~b}$ : Time history.

a)

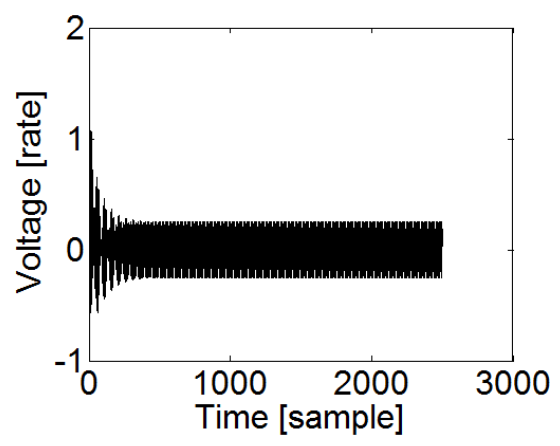

b)

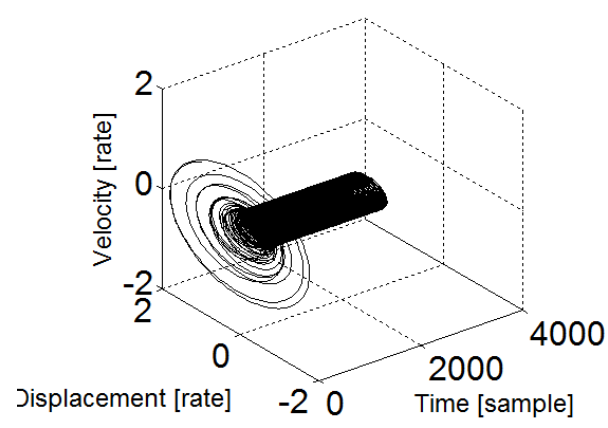

Figure 3: Dynamical behavior of the energy harvesting system.

\section{Controller Project}

An Optimal Linear Control (OLC) is defined to improve system efficiency, which guarantees the linear control application in non-linear systems. Considering a controlled non-linear system given by [1]:

$$
\dot{y}=A(t) y+h(y)+B u, \quad y(0)=y_{0}
$$


5 were $y \in \Re^{n}$ is a state vector, $A(t) \in \mathfrak{R}^{n x n}$ is a boundary conditions matrix (parameters) which elements are time depending, $B \in \Re^{n x m}$ is a matrix of constants, $u \in \Re^{m}$ is a control vector and $h(y) \in \mathfrak{R}^{n}$ is a vector which the elements are continuous non-linear functions, $h(0)=0$. It is highlighted that the chosen of $A(t)$ is not the only influence of controller efficiency. For a finite time interval and $A, B, Q$ e $\mathrm{R}$ been matrixes of constants elements, the positive defined matrix $\mathrm{P}$ is the solution of the algebric non-linear Riccati equation, dada given by:

$$
P A+A^{T} P-P B R^{-1} B^{T} P+Q=0
$$

Applying the OLC controller to the defined system the controller produces a gain vector $G=\left[\begin{array}{lllll}0.1346 & -0.1046 & 0.1000 & -0.1356 & -0.0082\end{array}\right]$ and consequently applying the equation $A_{c}=A-B G$, were $A$ is the original state matrix $A_{c}$ is the controlled state matrix, $B$ is the actuation vector and $G$ is the result gain vector, it is possible to determine the controlled system parameters as shown in Table 1 . The controlled system is also stable as all eigenvalues do have real part negative.

Table 1: Controlled system parameters.

\begin{tabular}{|c|c|}
\hline$\zeta$ & $1.0000 \mathrm{e}-04$ \\
\hline$\chi$ & 0.0582 \\
\hline$\Lambda$ & 0.0418 \\
\hline$\kappa$ & 0.3954 \\
\hline
\end{tabular}

Applying Runge-Kutta forth order in equation (4) for dimensionless parameters $\zeta=$ $0.01, \Omega=0.8, \chi=0.05, \kappa=0.5, \Lambda=0.05$ and $f=0.083$ [2] for the system without control and the parameters given in Table 1 for controlled parameters and initial conditions $x_{1}(0)=1, \varphi(0)=1, \dot{x}_{1}(0)=0, \dot{\varphi}(0)=0$ and $v(0)=0$ it is determined the rate of voltage and time history considering samples a from 0 to 2,500 in interval of 0.1 totalizing 25,000 time sample according Figure 3a: output voltage and 3b: Time history

a)

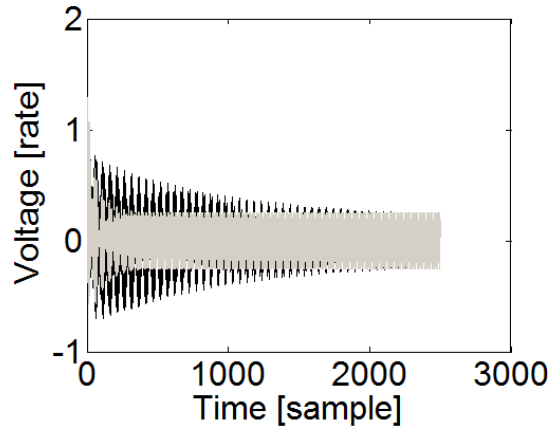

b)

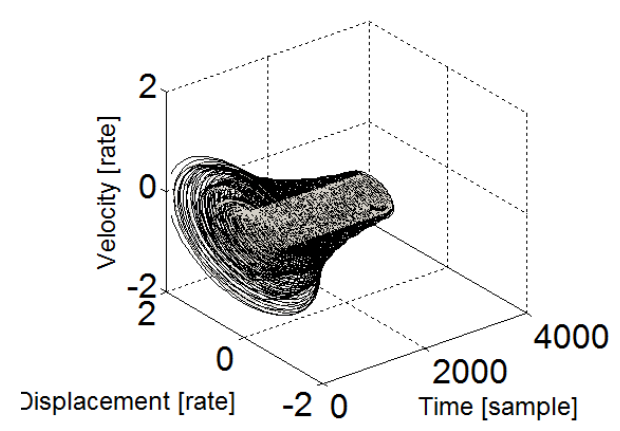

Figure 3: Efficiency gain via active control. 


\section{${ }^{6}$ Efficiency Gain Analysis}

As main result the controller increased the system efficiency in convert mechanical energy to electric output voltage. The Table 2 has shown the gain of efficiency via OLC active control according root mean square output voltage value.

Table 2: RMS voltage rate.

\begin{tabular}{|c|c|}
\hline Without control & 0.1927 \\
\hline OLC with LQR & 0.2292 \\
\hline Controller Gain & 1.1894 \\
\hline
\end{tabular}

\section{Conclusions}

As main conclusion the impact energy harvesting presented a feedback parameter that converts the system behavior from linear to nonlinear because a direction of movement of the driving beam to the generation beam. Is also highlighted that is possible to approximate the impacted system model to a non-ideal power source system.

As main conclusion applying OLC control to the system results an increase of 1.1894 times of output voltage from the system without control compared to the controlled system.

\section{Acknowledgements}

The authors would like to thanks to Federal University of Mato Grosso, Federal Technological University of Paraná, UNESP - Univ. Estadual Paulista for support. To Fundação de Amparo a Pesquisa do Estado de São Paulo (FAPESP Process No. 2014/16807-3) and Conselho Nacional de Desenvolvimento Científico e Tecnológico (CNPq Process No. 301769/2012-5) for funding. To Fundação Araucária, CAPES, FINEP, Fundação de Amparo a Pesquisa do Estado de Mato Grosso (FAPEMAT) and SETI for scholarship and funding.

\section{References}

[1] F. R. Chavarette, N. Peruzzi, J. M. Balthazar, L. Barbanti, B. C. Damasceno, On an optimal linear control applied to a non-ideal load transportation system, modeled whit periodic coefficients. Applied Mechanics and Materials, v. 52-54, 13-18 (2011), DOI: 10.4028/www.scientific.net/AMM.52-54.13.

[2] A. Erturk and D. J. Inman, Broadband piezoelectric power generation on high-energy orbits of the bistable duffing oscillator with electromechanical coupling, Journal of Sound and Vibration, v. 330, n. 10, 2339-2353 (2011), DOI: 10.1016/j.jsv.2010.11.018.

[3] D. C. Ferreira, F. R. Chavarette, N. J. Peruzzi, Optimal Linear Control Driven for Piezoelectric Non-Linear Energy Harvesting on Non-Ideal Excitation Sourced, Advanced Materials Research, v. 971-973, 1107-1112 (2014), DOI: 
7 [4] L. Gu, Low-frequency piezoelectric energy harvesting prototype suitable for the MEMS implementation, Microelectronics Journal, vol. 42, 277-282 (2011), DOI:10.1016/j.mejo.2010.10.007.

[5] R. L. Harne and K. W. Wang, A review of the recent research on vibration energy harvesting via bistable systems, Smart Mater. Struct., v. 22, 1-12 (2013), DOI: $10.1088 / 0964-1726 / 22 / 2 / 023001$.

[6] C. A. Howells, Piezoelectric energy harvesting. Energy Conversion and Management, v. 50, 1847-1850 (2009), DOI:10.1016/j.enconman.2009.02.020.

[7] M. Rafikov, J. M. Balthazar, On control and synchronization in chaotic and hyperchaotic system via linear control feedback, Communications in Nonlinear Science and Numerical Simulation, v. 13, 1246-1255 (2008), DOI: 10.1016/j.cnsns.2006.12.011

[8] S. Roundy, P. K. Wright, J. Rabaey, A study of low level vibrations as a power source for wireless sensor nodes, Computer Communications, v. 26, 1131-1144 (2003).

[9] I. Sari, T. Balkan, H. Kulah, An electromagnetic micro power generator for wideband environmental vibrations, Sensors and Actuators A, v. A156, 405-413 (2008), DOI: 10.1016/j.sna.2007.11.021.

[10] S. L. T. Souza, I. L. Caldas, J. M. Balthazar, R. M. L. R. F. Brasil, Characterization of non-ideal oscillators in parameter space. DINCON 2010, Serra Negra. 91-95 (2010), ISSN 2178-3667.

[11] L. Tang, Y. Yaowen, C. K. Soh, Broadband vibration energy harvesting techniques, Advances in Energy Harvesting Methods (Chapter Two). New York : Springer Science \& Business Media, 46p (2013).

[12] J. P. Thomas, M. A. Qidwai, J. C. Kellogg, Energy scavenging for small-scale unmanned systems. Journal of Power Sources, v. 159, 1494-1509 (2006), DOI: 10.1016/j.jpowsour.2005.12.084.

[13] A. M. Tusset, J. M. Balthazar, F. R. Chavarette, J. L. P. Felix, On energy transfer phenomena, in a nonlinear ideal and nonideal essential vibrating systems, coupled to a (MR) magneto-rheological damper, Nonlinear Dyn, 1-22 (2012), DOI:

[14] Y. Wang and J. D. Inman, A survey of control strategies for simultaneous vibration suppression and energy harvesting via piezoceramics. Journal of Intelligent Material Systems and Structures, v. 23, n. 18, 2021-2037 (2012), DOI: $10.1177 / 1045389 X 12444485$.

[15] W.-J. Wu, Y.-Y. Chen, B.-S. Lee, J.-J. He,Y.-T. Peng, Tunable resonant frequency power harvesting devices, Smart Structures and Materials, v. 6169, 1-8 (2006), DOI: $10.1117 / 12.658546$

[16] D. Zhu, M. J. Tudor, S. P. Beeby, Strategies for increasing the operating frequency range of vibration energy harvesters: a review.,Meas. Sci. Technol., v. 21, 1-29 (2010). DOI:10.1088/0957-0233/21/2/022001. 